\title{
Annotation management: a Group decision support system for nurses tasks
}

\author{
Philippe Marrast* and Pascale Zaraté \\ Laboratoire IRIT, Université de Toulouse, Toulouse, France \\ (Received 30 October 2014; accepted 12 March 2015)
}

\begin{abstract}
We develop a 5-year empirical investigation that is giving us broad and deep insights to characterise activity management in the palliative ward of an oncology hospital, and offer effective support for group decision-making and collaborative activity of caregivers. Following this observation period, we propose a software prototype based upon annotations in which dealing with patients' state and evolution is a complex organisational task. We based our conception of an annotation tool on the observations of the rich writing practices of medical professionals. We rely on the innovative strategy of intermediate management to introduce a new technology able to bridge heterogeneous, valuable data flows that addresses both management support and activity support in a single tool.
\end{abstract}

Keywords: annotations management; complex tasks; caregivers' coordination; data flows management; group activity

\section{Annotations to handle complex environments}

Our main research topic consists in the understanding of and computer support for organising processes in an uncertain, fast-changing and complex environment (Adla \& Zarate, 2006). We conducted empirical and qualitative research based on grounded theory methodology (Charmaz, 2006) and focused on nurses' practice of the everyday organisation of work (Bonneville \& Grosjean, 2007) in a palliative ward of an oncology hospital for more than 5 years.

This led us to consider annotation practice and annotative process (to be defined hereafter) as the core elements of the organisational work of caregivers in the ward to grab complexity and coordinate collective action in this highly evolving environment. We present in this paper a tool based on annotation management that we conceived for group decision-making and for the support of work organising practices.

In the second section of this paper, we will present the multidisciplinary state of the art about annotations management and about the relationships between writing practices, group decision-making and negotiated collective work. We will next develop our functional tool prototype for the organising work in oncology based upon annotations. And, finally, we will conclude and open discussion towards the opportunities and limits of such an approach to a collaborative decision support system.

*Corresponding author. Email: marrast@irit.fr 


\section{Annotations and their management: state of art}

Theoretically, we rely upon the Montreal school of text conversation models to address organisations and the organising process (Taylor \& Van Every, 2000). We also use Weick's concept of 'mindful interdependence' to interpret specifically the way caregivers mobilise and act into an interconnected network of human and non-human resources and actors to produce resilient and robust organisation despite organisational complexity (Weick, 2002). This theoretical frame is suitable to understand and analyse the intertwinement between text production, oral communication, organisation, technologies and patient management that we observe in our research ground.

\subsection{Methodological approach: user requirements analysis at the system design phase}

We did not literally work on a user requirements analysis in our design phase, not in the sense of system requirements engineering (Van Lamsweerde, 2009). Our study and model rely mostly upon empirical investigation based on ground observation of caregivers' practices. This approach gave us deep insights into the hospital organisation of work, caregivers' skills, coordination modalities and organising processes in wards, and so forth. This approach to system design is consistent both with participatory design to elicit and design from users' experience (reference) (Björgvinsson, Ehn, \& Hillgren, 2010; Gennari \& Reddy, 2000) and with grounded theory methodology (Charmaz, 2006).

Grounded theory intends to build theoretical and abstract models of social organisations, processes or individual/collective behaviours and experience. This methodology is an iterative process based on the progressive refinement of social models, that leads to abstract views of social worlds that we can translate into system design during the design process. Both theoretical and system models are continuously confronted with the real world they intend to model in order to gain robustness and become abstract and stable views of social realities.

Despite the benefits of such a user requirements method, the limits of this approach rest in the fact that these models are pictures of dynamic social organisations. These organisations are always on the move and are always adapting and rebuilding their social environment, their practices and their tools in order to adjust to the novelty of situations, the evolution of medical protocols, new regulations, constraints and so on. We have tried to integrate this dynamic feature of our case study into our prototype design that is intended to be adaptive to users' needs.

\subsection{From the functional view of annotations to a communicational and organisational perspective of annotations}

While acknowledging flexibility and ease of use, but also hermeneutic and heuristic abilities of annotations, scientific communities that are involved with these practicetools consider them, in all our readings, as a phenomenon. Annotation practice emerges due to complex environments, due to the 'lack' of memory of workers and due to the rigidity of formal and numeric documents. It is often pointed to as a pragmatic response to complexity but is never used as the core element of organising processes (Bringay, Barry, \& Charlet, 2006; Lewkowicz, Lortal, Todirascu, Zacklad, \& Sriti, 2004; Weng \& Gennari, 2004). 
Annotations are perceived as peripheral and secondary elements, the purpose of which is to enrich or interconnect texts or objects, to memorise temporary information or to remember something to do. Annotations have been often described as structuring tools at a micro-social level (Bringay et al., 2006; Lewkowicz et al., 2004). Their features enable them to support cooperation and coordination modalities of small collectives of work. Annotation considered as a practice allows caregivers to capture quickly and easily relevant events in organisation and care activity, to sort and synthesise elements (writer-/reader-dependent), to discuss specific elements during transmissions, to make hypotheses, to give instant access to these items through various medias (Bardram \& Bossen, 2005) and to distribute collective awareness and watchfulness between members of the collective.

Annotations have the ability to interconnect and integrate synthetically various data sources, and to assemble heterogeneous organisational elements (see Figure 1; e.g. Electronic Patient Record (EPR), Electronic Medical Record (EMR), oral transmissions, artefacts of the environment, scheduling, etc.). Annotations offer flexibility and ease of use that permit us to answer to the milfoil of action modalities, superposed temporal constraints (physicians visits, patients' care, patient entry or exit, etc.), distributed data and complexities of situations in a palliative ward (Bringay et al., 2006).

From the reader point of view, annotations embark on various 'functionalities': to-do lists, reminders, questions, interpretations, thoughts. They activate specific watchfulness thanks to the writing modality (colour, forms, underlining, 'anchor'). Annotation is a powerful tool used to characterise, to remind, to coordinate, to develop

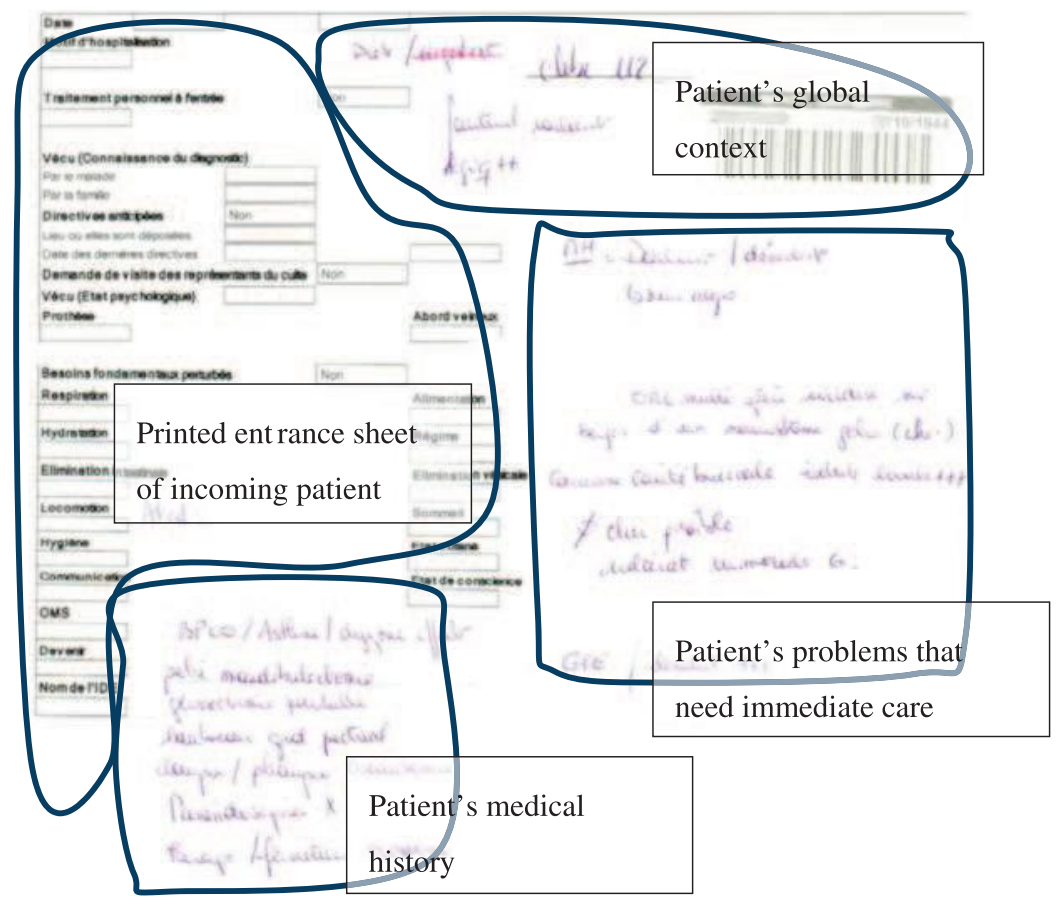

Figure 1. The annotative practice and patient representation practice: a nurse printed a patient entrance form (heuristic flow), added the room number and stuck patient barcode (institutional flow) and added handwritten notes with data extracted from EMR (institutional flow) plus personal notes in order to plan further action (interview of patient and gathering of up to date data). Note: EMR: Electronic Medical Record. 
aboutness and, finally, to manage patients' care context that contains many interwoven dimensions: medical, social, temporal, technical, cognitive, regulatory, organisational, and so on (Lewkowicz et al., 2004, Reddy, Dourish, \& Pratt, 2006).

From an organisational point of view, the annotation practice that we observe in our research ground can be seen as a cultural practice that structures and configures the organisation of care work as much as it shapes the sociotechnical collectives (Berg, 1999). As annotations are extracted from patients' context, negotiated and then put back in the patients' history and traced into Health Information System (HIS) bricks, they co-configure writing practices, as much as they are part of the group decision process as involved and 'agentive' actors (Mayère, Bazet, \& Roux, 2011; Reddy \& Jansen, 2008).

Finally, annotations can be seen as micro stories that are told and re-told many times a day by different spokesmen in order to verify every piece of information about patients. It is a very collective work of informational forging which gives consistency to data, to patients' stories and trajectories, and to group decisions that validate or invalidate parts of or sometimes the entire therapeutic plan.

\subsection{Annotations as the core of organising processes: the annotative practice}

Despite the huge number of intrinsic qualities of annotations that our literature review and our ground observations point out, still the annotations are considered as secondorder elements, useful but not key objects of concern for group decision support or the Computer Supported Cooperative Work (CSCW) field. Now, we will formulate the hypothesis that annotations can be considered opportunely as constitutive elements not peripheral - in the production of documents and in the forging of organisational texts. Beyond documentary features, we will also characterise them as elementary bricks that are constitutive elements in the organisation of medical work that is closely tied to text production, that intervene in managing patients' pathology, trajectory and care (Berg, 1999; Mayere, Bazet, \& Roux, 2012; Star \& Strauss, 1999). Annotations are core elements of the everyday practice of organising in an oncology ward. Caregivers rely on what we could call an 'annotative practice' to handle their complex environment of work and the complex situations of patients they take care of. This annotative practice deals with three valuable data flows (see Figure 2):

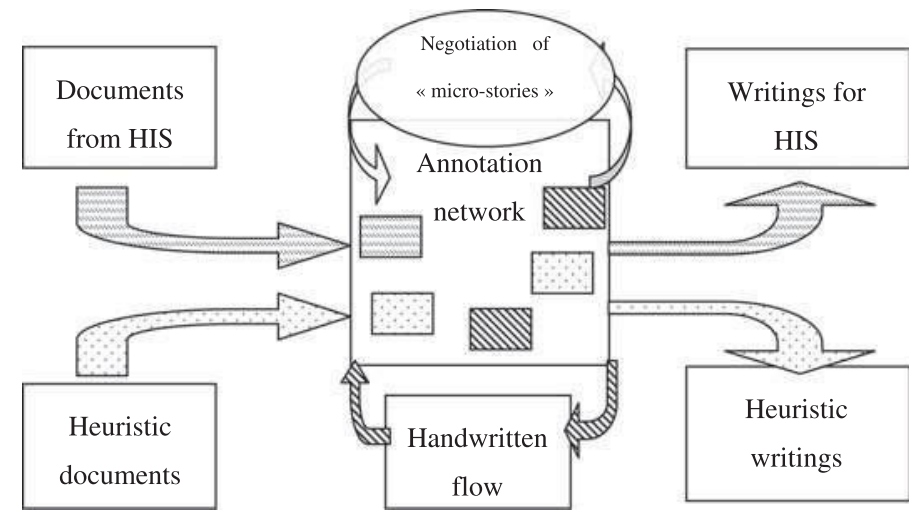

Figure 2. Valuable flows and annotative practice cycle: documents are split into manageable pieces connected to each other (author, subject or target). Each piece is negotiated during team shift transmissions. Negotiated pieces are stored into a collective form and the cycle restarts.

Note: HIS: Health Information System. 
- The institutional flow mediated by EPR and EMR. This flow is impelled by professional regulatory constraints. It is often composed of a huge number of exhaustive technical documents related to patients' history, disease, laboratory tests, imagery, etc. This flow is difficult to handle and use in everyday activity due to the encyclopaedic view of patients that it provides. Hospital organisations risk financial penalties if the quality and completeness of this flow is insufficient with regard to the law.

- On the other side of valuable document flows, we observe personal writings or very small collective writing flows, mediated by printed sheets heavily annotated during work. This flow can also be embedded in various artefacts (drug packaging, sticky notes). This is the core flow of what we call the annotative practice. Caregivers literally rebuild a very rich and situated set of data to grab the world, understand the situations and act into the complex environment of the palliative ward. This flow has two main inconveniences. It is hard to normalise due to the personal practice of writing, and it is produced outside HIS.

- The third flow we were able to observe is the result of an innovation driven by intermediate management of the palliative ward in order to articulate the two valuable flows depicted above. The caregivers in the ward developed iteratively a collective flow mediated by heuristic documents. This flow offers a synthetic view of all the patients in the ward in two pages of A4 format. This text sheet is managed by the nurses of all the teams with a standard text editor and stored in a local file on the ward computer. This document is printed and annotated during work and used for oral transmission during team shifts.

These three valuable data flows act in different layers of the organisation (institutional, collective, individual), but they are not independent from each other. Each flow is correlated to the others and produces either a frame or a complement to the other flows. These flows help caregivers in awareness and decision-making, for therapeutic adjustment and for the articulation and coordination of and cooperation in the realisation of the multiple and complex tasks they have to carry out to take care of patients. These flows are all melted together thanks to document manipulation, and during team shift transmission. As these flows are produced by heterogeneous sources and contain both redundant and complementary data, caregivers normalise data flows by what we call an annotative practice. Caregivers use a sort of pivot format, namely annotations, to mix and reshape heterogeneous data for their individual and collective purposes, and then put data back into the right destination flow.

Therefore, we consider that organising work is correlated to communication processes mediated by and embedded in writing practices that are co-constitutive of organisation (Taylor \& Van Every, 2000) and which produce a network of mindful interdependence (Weick, 2002).

Our goal in the design of our prototype is to rely upon caregivers' innovative practice and to articulate these three valuable flows that address various organisational requirements into a single tool based on annotations.

\section{From empiric approach to software prototyping}

Taking a look at tools such as social networks, participative conception tools, workflow modelling systems or ERP, we noticed that users have the ability to define models of activity, plan actions, lists and organise tasks and tell stories. But until now, the layer of 
organising as we have presented is often neglected. Tools on shelves are often proposed as a 'system as is' from a requirements engineering perspective (Van Lamsweerde, 2009).

Our empirical investigation and our literature review taught us that in everyday situations, technical systems are both system as is and system to be. Tools shape practices and they are shaped by the repeated practices and experiments of caregivers (Ash, Berg, \& Coiera, 2004; Berg, 1997). The only 'independent variable' that we were able to point out is in fact the annotative practice that we have just described. So, in order to reach the needs of caregivers and the requirements of standards, HIS and regulatory constraints, we need to go beyond the limitations of these tools and standards (Briggs, Kolfschoten, de Vreede, Albrecht, \& Lukosch, 2010). This is why we populate the organising layer with 'smart' annotations that reproduce writing practices and documentarisation practices of caregivers and this is also why we develop a prototype which is a 'system to be' that caregivers can design by themselves. The system can be seen as a toolbox that supports collaboration in activity. We allowed all kinds of users to use one view of the system. They can then update all other views, allowing a global apprehension of the group activity.

Thanks to its functionalities, our tool can participate in both institutional and ambient organising, offering flexibility and instant access, ease of use and, more importantly, robustness and resilience for the organisation of medical activity. This overlay allows caregivers to articulate heterogeneous sources of data into a single 'blender' in order to build a situated informational system connected to and interoperable with the institutional one. To address these issues, our tool provides three main functionalities. It first gives the ability to split documents of all kinds into an annotation network with references to the original document. Then caregivers can manage the organisation of work and data through a connected network of 'smart' extended and connected annotations. Finally, they can create composite tools above the annotation layer to give usable shapes to the annotation network.

An important element of this tool is the multi-view system. One annotation can be seen in several views - for example:

- 'Mindmap' view (Figure 3): This is the default view, which is useful in order to organise topics visually (size, colour, drawings, etc.) and geographically on the screen;

- Heuristic view (Figure 4): This view has been inspired by our investigation. In this view, nurses can synthesise patients' state, pathology, medical history and so forth. This view is the main tool that nurses use during activity or during team shifts in order to share and actualise data about patients;

- Project management view (Figure 5): Annotations can be tagged with beginning and end dates or with scheduling features (date, repetition patterns, etc.), and resources can be attached to annotations; therefore, they can be used as elementary bricks of project management (i.e. folders, tasks, reminders, resources, etc.);

- Tasks to-do list (Figure 6): This view is also called the 'agile' view in reference to the agile scrum board that allows nurses to build and visualise an ongoing collective activity and a shared understanding of the care to give;

- Structured document view: As annotations are imbricated in one another, they can be used as structured text containers usable for building documents, and can be exported in a sharable form (Word, pdf, open document format, etc.);

- Grid view: A view in which annotations are organised via a grid. This feature is useful for managing folders, pictures or videos libraries, etc. 


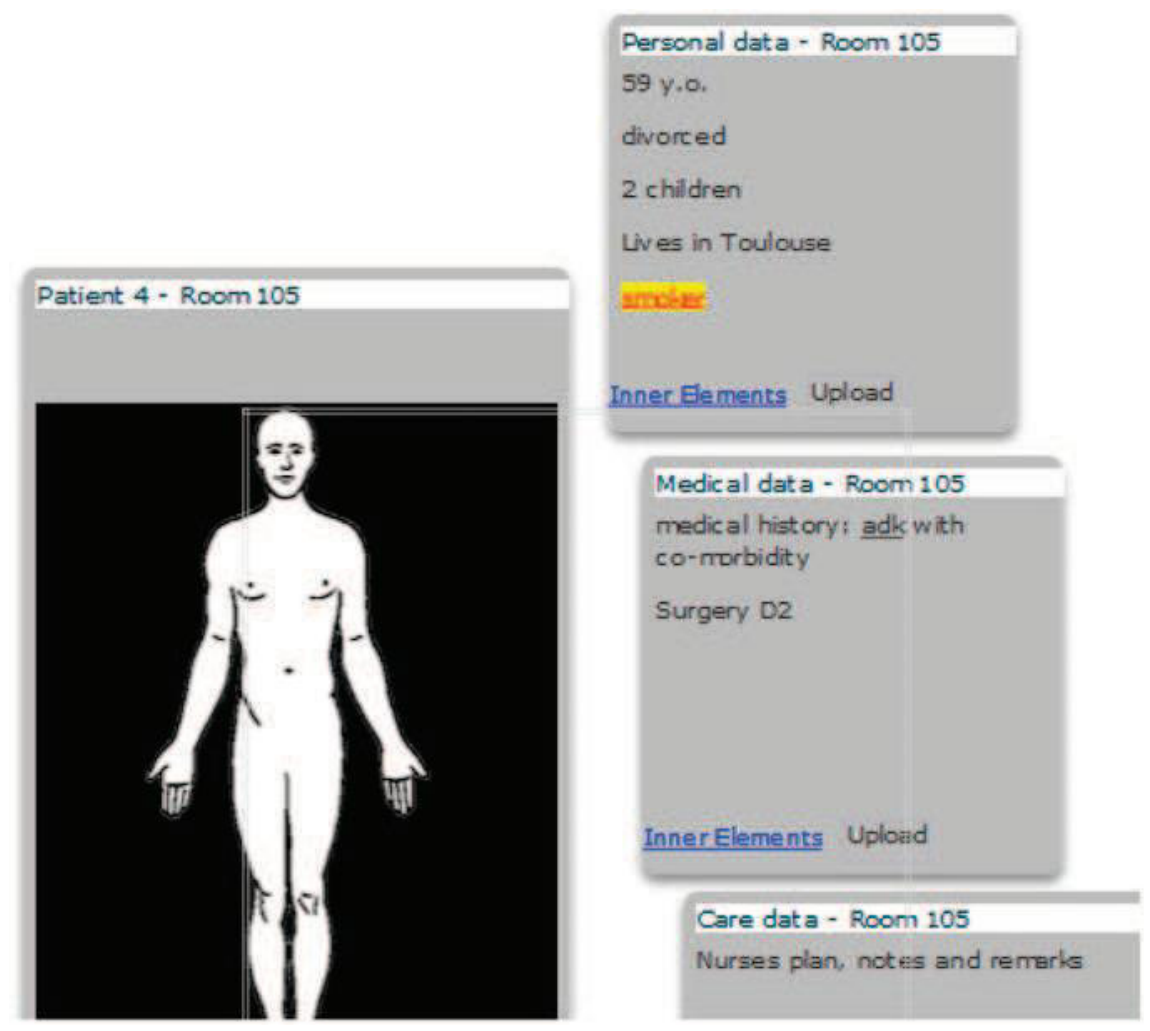

Figure 3. Mindmap view.

\begin{tabular}{|c|c|c|c|c|}
\hline patient's context & medical history & problematics & equipment & nursing \\
\hline $\begin{array}{l}59 \text { yo. } \\
\text { divorced } \\
2 \text { chidren } \\
\text { Lives in Toulouse }\end{array}$ & $\begin{array}{l}\text { adk with co-morbidity } \\
\text { Surgery D2 }\end{array}$ & $\begin{array}{l}\text { Nurses plan, } \\
\text { notes and remarks }\end{array}$ & $\begin{array}{l}\text { Nurses often list } \\
\text { specific equipment } \\
\text { for patients }\end{array}$ & \\
\hline & & & & \\
\hline & & & & \\
\hline & & & & \\
\hline
\end{tabular}

Figure 4. Heuristic view for patient global awareness and collective sharing.

The system is thus based on a database of annotations that can be manipulated and then shaped for adequate further handling (presentation, documentation, project and task management, scheduling, etc.). 


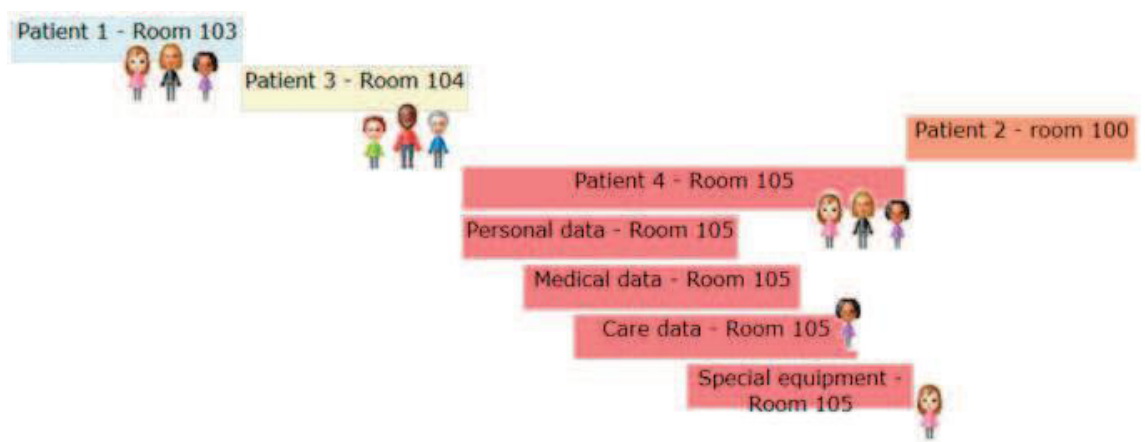

Figure 5. Gantt view.

\begin{tabular}{|c|c|c|c|c|}
\hline todo & doing & testing & validating & done \\
\hline $\begin{array}{l}\text { Patient } \\
2 \\
\text { Patient } \\
4-12\end{array}$ & $\begin{array}{c}\text { Patient } \\
3 \\
3\end{array}$ & $\begin{array}{c}\text { Patient } \\
{ }^{1} \mathrm{~A}\end{array}$ & & \\
\hline
\end{tabular}

Figure 6. 'Agile' view of patients with instant access to ongoing work and resource allocation.

\subsection{Annotation modelling}

Annotation is the core object of our prototype. In other words, all the objects and classes related to data-flow management are extended from the annotation class; annotation is the constitutive class of our application.

As annotations have the ability to transform objects into a single pivot format of data, we have to design them to be compatible with the usual objects of writing practices and organising and communicational processes. For example, we equip our annotations with event management features (begin/end date, repetition, etc.), messaging features (author/sender, recipients, attachments) or search/auto-completion abilities.

The following is a non-exhaustive list of features of these smart annotations (Figure 7).

\subsection{Editing abilities of annotations}

- Annotations can handle content style layout (thanks to a wysiwyg editor);

- Annotations support drawings (through a svg editor);

- They have an auto-complete feature which can connect them to external nomenclature (medical, equipment, patient or caregiver directory, etc.); 


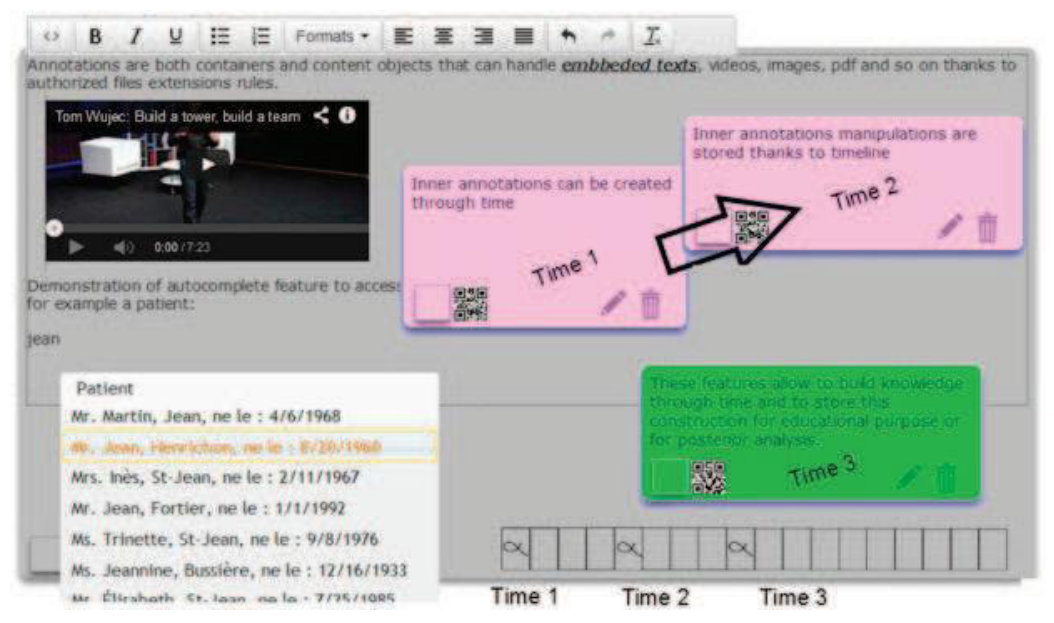

Figure 7. Screenshot of annotations that illustrates some of their smart functionalities: multimedia container, cross reference preservation and auto-completion. Inner 'timeline' holds synchronous or asynchronous updates of annotation content (versionChild) and inner annotations state.

- They have a spell-checker functionality that allows caregivers to access various dictionaries, generate a pop-up glossary and handle local vocabulary (in order to build local acronyms and maintain ontological reference to a term).

\subsection{Containing abilities}

- Multimedia container ability (text, html, images, videos, attached content);

- Self-containing: an annotation can hold and be held in an annotations net;

- Have an inner timeline that manages internal states evolutions;

- Supports multi-authoring.

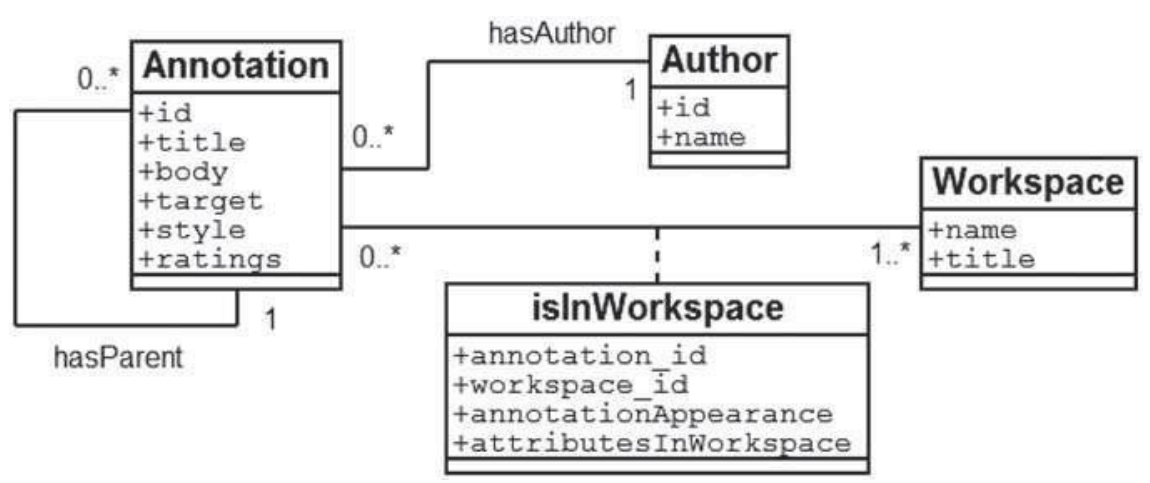

Figure 8. Simplified annotation class diagram. 


\subsection{Annotation model characteristics and class diagram}

We rely on the work of the Open Annotation Collaboration (OAC) workgroup paradigm for our annotations model. We use this general frame for our annotation model in order to be compliant with web standards of connected objects and medical document standards (HL7) to prepare our prototype for further integration (Figure 8).

Our contribution to these standards stands in the fact that our annotations embark on versioning, rich media management, 'smart' data contextualisation through nomenclature and dictionary connections, and an internal timeline.

\section{Perspectives and conclusion}

Before concluding this paper, there are questions brought to us by reviewers that we want to discuss as potential and insightful perspectives on our work.

How is the interest of the functionalities measured?

How is the completeness of the developed model measured?

The question of design acceptability and integration in the research field we investigate is a sensitive one for our work. Unlike many other projects in group decision support, our contribution is not an order from the field. Our conclusions are less driven by expected results than they are by methodological stance. Therefore, our tool was never intended to replace or improve existing ones. It was conceived so that it could reflexively inform with an external eye and help the management and the stakeholders in the hospital to understand and perhaps rebuild more accurately their organisational processes.

We have integrated this constraint in our iterative design. To do so, we conducted some focus groups during different stages of our prototype design with management staff and matrons. We organised three of these meetings in one year, each of which lasted a couple of hours. Each time, we had in-depth analysis of our prototype with interactions with people, audio recording and video each time we could.

These meetings helped us in two ways. They clearly helped us out with issues regarding our understanding of organisational processes and practices, but they also confronted in a very insightful way the management view of ward life and organisation with the nurses' view that we observed during our investigations and which we translated into our prototype.

One of the main lessons of this - sometimes unexpected - confrontation is the fact that organising processes and group decision-making cannot be reduced - whatever the method or the accuracy - into a single translation. This major point brings us to the second part of the reviewers' questions concerning the completeness of our model.

As was pointed out by Sinha, Sunder, Bendale, Mantri, and Dande (2012), medical computing standards embrace the notion of intrinsic 'systems to be'. The variety of situations, modalities of work and cooperation, the variable and permeable boundaries between professional skills, roles and missions, or the various national regulations of medical work and public health policy, etc. are parameters and possible model instantiations to consider when designing a decision support system for medical work.

Facing such a complexity, our annotation model has an inner strength and robustness: its simplicity. Our tool is very simple to manipulate, apprehend, use and extend from the basis we have created. But despite this simplicity, the question of completeness is still relevant and open. 
The annotation model we have just proposed is the first step of our work. The challenge we face now is to design a sort of DIY layer upon our annotation model that will allow users to autonomously develop their own tools based upon annotations web. We do believe that this design to come will be able to bridge and to articulate the numerous views, practices, regulatory issues and medical standards into a single tool.

This last part of our project will not close the question of the completeness of our model, but it will open oppositely the perspective of emergences of models and forms and the issue of adaptability to medical practice complexities.

In conclusion, we proposed in this paper a new paradigm for group decisionmaking and collaborative work support: the constitutive role of annotations to address organisational complexity and manage heterogeneous valuable data flows.

Our contribution to the domain lies in the proposal of enhanced functionalities of annotations based on deep observation of writing practices. Our proposal argues that translating this practice into a software design is full of interesting potentialities for the group decision support domain. This allows us to maintain a structuring, organising and constitutive practice, and to build robustness and resilience by the intensive everyday usage of writing tools and the co-creation process that we observed (Hartswood et al., 2003).

The stake in our proposal is to go beyond annotation tools as a collaborative tool for collective writing. We propose to equip classical HIS with a layer of organising based upon annotations to manage valuable flows of data and co-create organising and mindful interdependence. This construction could help caregivers to build a culture of interoperable writings that match both activity needs and normative standards of medical documents. Hence, our tool could help to build a richer and extended writing culture towards the professionalisation of medical writings.

This prototype is a first step in a more complex development process. This system aims to support nurses' activity but is general enough to be used by any kind of team for whom coordination is a necessary part of their activity.

\section{Disclosure statement}

No potential conflict of interest was reported by the authors.

\section{References}

Adla, A., \& Zarate, P. (2006). A cooperative intelligent decision support system. In Service Systems and Service Management, 2006 International Conference on (Vol. 1, pp. 763-769), Troyes, France. http://doi.org/10.1109/ICSSSM.2006.320558

Ash, J. S., Berg, M., \& Coiera, E. (2004). Some unintended consequences of information technology in health care: The nature of patient care information system-related errors. Journal of the American Medical Informatics Association: JAMIA, 11, 104-112. http://doi.org/10. 1197/jamia.M1471

Bardram, J. E., \& Bossen, C. (2005). A web of coordinative artifacts: Collaborative work at a hospital ward. In Proceedings of the 2005 international ACM SIGGROUP conference on Supporting group work (pp. 168-176), Sanibel Island, Florida.

Berg, M. (1996). Practices of reading and writing: The constitutive role of the patient record in medical work. Sociology of Health \& Illness, 18, 499-524. http://doi.org/10.1111/1467-9566. ep10939100

Berg, M. (1997). Of forms, containers, and the electronic medical record: Some tools for a sociology of the formal. Science, Technology \& Human Values, 22, 403-433. http://doi.org/ $10.1177 / 016224399702200401$ 
Berg, M. (1999). Patient care information systems and health care work: A sociotechnical approach. International Journal of Medical Informatics, 55, 87-101.

Björgvinsson, E., Ehn, P., \& Hillgren, P. A. (2010). Participatory design and democratizing innovation. In Proceedings of the 11th Biennial Participatory Design Conference (pp. 41-50), Sydney, Australia.

Bonneville, L., \& Grosjean, S. (2007). Les défis que soulève l'informatisation de la pratique médicale sur le plan de l'innovation technologique [The challenges raised by computerization of medical practice in terms of technological innovation]. Canadian Journal of Communication, 32 .

Briggs, R. O., Kolfschoten, G. L., de Vreede, G.-J., Albrecht, C. C., \& Lukosch, S. G. (2010) Facilitator in a box: Computer assisted collaboration engineering and process support systems for rapid development of collaborative applications for high-value tasks. In System Sciences (HICSS), 2010 43rd Hawaii International Conference on (pp. 1-10). IEEE. Retrieved from $\mathrm{http}: / /$ ieeexplore.ieee.org/xpls/abs all.jsp?arnumber $=5428697$

Bringay, S., Barry, C., \& Charlet, J. (2006). Annotations: A functionality to support cooperation, coordination and awareness in the electronic medical record. In Proceeding of the 2006 conference on Cooperative Systems Design: Seamless Integration of Artifacts and Conversations-Enhanced Concepts of Infrastructure for Communication ( $\mathrm{pp}$. 39-54), Amsterdam, The Netherlands.

Charmaz, K. (2006). Constructing grounded theory: A practical guide through qualitative analysis. New York, NY: Pine Forge Press.

Gennari, J. H., \& Reddy, M. (2000). Participatory design and an eligibility screening tool. In Proceedings of the AMIA symposium (p. 290). American Medical Informatics Association, Los Angeles, CA.

Hartswood, M., Procter, R., Rouchy, P., Rouncefield, M., Slack, R., \& Voss, A. (2003). Working IT out in medical practice: IT systems design and development as co-realisation. Methods of Information in Medicine, 42, 392-397.

Lewkowicz, M., Lortal, G., Todirascu, A., Zacklad, M., \& Sriti, M. F. (2004). A web-based annotation system for improving cooperation in a care network. In ICWE Workshops (pp. 227-239).

Mayere, A., Bazet, I., \& Roux, A. (2012). "Zéro papier » et « pense-bêtes » à l'aune de l'informatisation du dossier de soins ["Paperless" and "reminders" in the light of computerization of the patient care record]. Revue D'anthropologie Des Connaissances, 6, 1, 115-139.

Mayère, A., Bazet, I., \& Roux, A. (2011). The agency of information, machines and instruments in sensemaking and cooperative work: The case of computerised patient records and paper reminders. The Communicative and Material Constitution of Organization. Studying Objects, Texts and Sites, $61^{\text {st }}$. Boston, MA.

Reddy, M. C., \& Jansen, B. J. (2008). A model for understanding collaborative information behavior in context: A study of two healthcare teams. Information Processing \& Management, 44, 256-273.

Reddy, M. C., Dourish, P., \& Pratt, W. (2006). Temporality in medical work: Time also matters. Computer Supported Cooperative Work (CSCW), 15, $29-53$.

Sinha, P. K., Sunder, G., Bendale, P., Mantri, M., \& Dande, A. (2012). Electronic health record: Standards, coding systems, frameworks, and infrastructures (1st ed.). Hoboken, NJ: Wiley-IEEE Press.

Star, S. L., \& Strauss, A. (1999). Layers of silence, arenas of voice: The ecology of visible and invisible work. Computer Supported Cooperative Work (CSCW), 8, 9-30.

Taylor, J. R., \& Van Every, E. J. (2000). The emergent organization: Communication as its site and surface. Mahwah, NJ: Lawrence Erlbaum.

Van Lamsweerde, A. (2009). Requirements engineering - from system goals to UML models to software specifications. Hoboken, NJ: Wiley.

Weick, K. E. (2002). The reduction of medical errors through mindful interdependence. In M. M. Rosenthal \& K. M. Sutcliffe (Eds.), Medical error: What do we know? What do we do (pp. 177-199), San Francisco: Jossey-Bass.

Weng, C., \& Gennari, J. H. (2004). Asynchronous collaborative writing through annotations. In Proceedings of the 2004 ACM conference on Computer supported cooperative work (pp. 578-581). ACM. Retrieved from http://dl.acm.org/citation.cfm?id=1031705 